\title{
THE PROPER CLASS GENERATED BY WEAK SUPPLEMENTS
}

\author{
Rafail Alizade$^{1}$, Yılmaz M. Demirci ${ }^{2}$, Yılmaz Durğun², \\ and Dilek Pusat ${ }^{2}$ \\ ${ }^{1}$ Department of Mathematics, Yaşar University, Selçuk Yaşar Campus, \\ Bornova, Izmir, Turkey \\ ${ }^{2}$ Department of Mathematics, Izmir Institute of Technology, Urla, \\ Izmir, Turkey
}

We show that, for hereditary rings, the smallest proper classes containing respectively the classes of short exact sequences determined by small submodules, submodules that have supplements and weak supplement submodules coincide. Moreover, we show that this class can be obtained as a natural extension of the class determined by small submodules. We also study injective, projective, coinjective and coprojective objects of this class. We prove that it is coinjectively generated and its global dimension is at most 1. Finally, we describe this class for Dedekind domains in terms of supplement submodules.

Key Words: Coatomic modules; Coatomic supplement submodule; Coinjective modules; Coprojective modules; Extended weak supplement; Proper class of short exact sequences; Weak supplement submodule.

2010 Mathematics Subject Classification: Primary 18G25; Secondary 13C60, 16D90.

\section{INTRODUCTION}

Proper classes were introduced by Buchsbaum to axiomatize conditions under which a class of short exact sequences of modules can be computed as Ext groups corresponding to a certain relative cohomology. It is well-known that the class $S$ uppl of short exact sequences determined by supplement submodules is proper in this sense of Buchsbaum (see [4, 20.7]). However, many other analogous classes of short exact sequences of modules do not form a proper class. For instance, in this article we are interested in the classes $\mathscr{S}$ mall, $\mathscr{S}$, and $\mathscr{W} \mathscr{S}$ determined by small submodules, submodules that have supplements and weak supplement submodules respectively. These classes are not proper in general, but for each of them we may consider the smallest proper class containing them, that is, the intersection of all proper classes containing them. We show that, for a hereditary ring $R$, they generate the same proper class $\overline{\mathscr{W} S}$ and this proper class can be obtained as a natural extension of $\mathbb{W S}$.

Received March 9, 2011; Revised October 20, 2011. Communicated by J. L. Gomez Pardo.

Address correspondence to Dr. Dilek Pusat, Department of Mathematics, Izmir Institute of Technology, Gülbahçeköyü, Urla, Izmir 35430, Turkey; E-mail: dilekyilmaz@iyte.edu.tr 
Once we have identified $\overline{\mathscr{W S}}$, we study in Section 4 , its most significative objects: injective, projective, coinjective, and coprojective objects. We first note that injective and projective objects in this class coincide with the injective and projective objects in $\mathscr{S}$ mall, $\mathscr{S}$, and $\mathscr{W} \mathscr{S}$ (see [10]). And then, we prove that $\overline{\mathscr{W} S}$ is coinjectively generated, namely, it is the smallest proper class for which every small module is coinjective. Now we can apply a dual argument to the proposition in [2, p. 181] to obtain gl.dim $\overline{\mathscr{W S}} \leq 1$ over a hereditary ring. Finally, in Section 5, we also describe the class $\overline{\mathscr{W S}}$ in terms of supplement submodules: we show that $A$ is a $\overline{\mathscr{W S}}$ submodule of $B$ if and only if there is a submodule $C$ of $B$ such that $A+C=B$ and $A \cap C$ is coatomic.

Any unexplained material is standard, as in [1].

\section{PRELIMINARIES}

Let $R$ be an associative ring with identity and $\mathscr{P}$ be a class of short exact sequences of left $R$-modules and $R$-module homomorphisms. If a short exact sequence

$$
E: 0 \longrightarrow A \stackrel{f}{\longrightarrow} B \stackrel{g}{\longrightarrow} C \longrightarrow 0
$$

belongs to $\mathscr{P}$, then $f$ is said to be a $\mathscr{P}$-monomorphism and $g$ is said to be a $\mathscr{P}$-epimorphism. A short exact sequence $E$ is determined by each of the monomorphisms $f$ and the epimorphisms $g$ uniquely up to isomorphism.

Definition 2.1. The class $\mathscr{P}$ is said to be proper (in the sense of Buchsbaum) if it satisfies the following conditions [7]:

P-1) If a short exact sequence $E$ is in $\mathscr{P}$, then $\mathscr{P}$ contains every short exact sequence isomorphic to $E$.

P-2) $\mathscr{P}$ contains all splitting short exact sequences.

P-3) The composite of two $\mathscr{P}$-monomorphisms is a $\mathscr{P}$-monomorphism if this composite is defined.

P-3') The composite of two $\mathscr{P}$-epimorphisms is a $\mathscr{P}$-epimorphism if this composite is defined.

P-4) If $g$ and $f$ are monomorphisms, and $g \circ f$ is a $\mathscr{P}$-monomorphism, then $f$ is a $\mathscr{P}$-monomorphism.

P-4') If $g$ and $f$ are epimorphisms, and $g \circ f$ is a $\mathscr{P}$-epimorphism, then $g$ is a $\mathscr{P}_{-}$ epimorphism.

We will identify a class of isomorphic short exact sequences with any of its elements. The set $\operatorname{Ext}_{\mathscr{g} P}^{1}(C, A)$ of all short exact sequences of $\operatorname{Ext}^{1}(C, A)$ that belongs to a proper class $\mathscr{P}$ is a subgroup of the group of the extensions $\operatorname{Ext}_{R}^{1}(C, A)$. Conversely, given a class $\mathscr{P}$ of short exact sequences, if $\operatorname{Ext}_{\mathscr{P}}^{1}(C, A)$ is a subfunctor of $\operatorname{Ext}_{R}^{1}(C, A), \operatorname{Ext}_{\mathscr{P}}^{1}(C, A)$ is a subgroup of $\operatorname{Ext}_{R}^{1}(C, A)$ for every $R$-modules $A, C$, and if the composition of two $\mathscr{P}$-monomorphisms (or $\mathscr{P}$-epimorphisms) is a $\mathscr{P}_{-}$ monomorphism (a $\mathscr{P}$-epimorphism respectively), then $\mathscr{P}$ is a proper class (see Theorem 1.1 in [9]). 
For any class $\mathscr{E}$ of short exact sequences the intersection of all proper classes containing $\mathscr{E}$ is clearly a proper class and it is denoted by $\langle\mathscr{E}\rangle$. We say that $\langle\mathscr{E}\rangle$ is the proper class generated by $\mathscr{E}$ (see [10]). Clearly, $\langle\mathscr{E}\rangle$ is the smallest proper class containing $\mathscr{E}$. A module $M$ is called Ë-projective (respectively Ë-injective) if the sequence $\operatorname{Hom}(M, E)$ (respectively $\operatorname{Hom}(E, M)$ ) is exact for every $E$ from $\mathscr{E}$. The class of all $\mathscr{E}$-projective (respectively $\mathscr{E}$-injective) modules is denoted by $\pi(\mathscr{E})$ (respectively by $l(\mathscr{E})$ ). The following proposition shows that the proper class $\langle\mathscr{E}\rangle$ generated by $\mathscr{E}$ has the same projective and injective modules as $\mathscr{E}$.

Proposition 2.2 ([10, Propositions 2.3 and 2.4]).

(a) $\pi(\mathscr{E})=\pi(\langle\mathscr{E}\rangle)$.

(b) $\imath(\mathscr{E})=\imath(\langle\mathscr{E}\rangle)$.

A module $M$ is called $\mathscr{P}$-coprojective (respectively $\mathscr{P}$-coinjective) if every short exact sequence of the form $0 \longrightarrow A \longrightarrow B \longrightarrow M \longrightarrow 0$ (respectively, $0 \longrightarrow M \longrightarrow$ $B \longrightarrow C \longrightarrow 0$ ) is in $\mathscr{P}$. Let $M$ and $\mathscr{g}$ be classes of modules over some ring $R$. The smallest proper class $\bar{k}(M)$ such that all modules in $M$ are $\bar{k}(M)$-coprojective is said to be coprojectively generated by $M$. The proper class $\underline{k}(\mathscr{f})$ which is coinjectively generated by $g$ is defined dually.

For a homomorphism $f: A \longrightarrow B$ and a module $C$ the induced homomorphisms $\operatorname{Ext}^{1}\left(1_{C}, f\right): \operatorname{Ext}^{1}(C, A) \longrightarrow \operatorname{Ext}^{1}(C, B)$ and $\operatorname{Ext}^{1}\left(f, 1_{C}\right)$ : $\operatorname{Ext}^{1}(B, C) \longrightarrow \operatorname{Ext}^{1}(A, C)$ we will denote by $f_{*}$ and $\operatorname{Ext}^{1}(f, 1)$ by $f^{*}$ as in [7, Chapter 3, Section 2]. The following Theorem follows from [1, Theorem 2].

Theorem 2.3. If $\mathscr{F}$ is a class of modules closed under extensions, then $\underline{k}(\mathscr{f})$ consists of all short exact sequences $f_{*}(E)$ where $E$ is a short exact sequence of the form $E$ : $0 \longrightarrow J \longrightarrow B \longrightarrow C \longrightarrow 0$ with $J \in \mathcal{F}$ and $f: J \rightarrow A$ is a homomorphism.

Considering long exact sequences consisting only of $\mathscr{P}$-monomorphisms and $\mathscr{P}$-epimorphisms one can define $\operatorname{Ext}_{\mathscr{P}}^{n}(C, A)$ (see [7]).

Definition 2.4. For a proper class $\mathscr{P}$ of short exact sequences of $R$-modules, the global dimension of $\mathscr{P}$ is defined as

$$
\text { gl.dim } \mathscr{P}=\inf \left\{n \mid \operatorname{Ext}_{\mathscr{P}}^{n+1}(C, A)=0 \text { for all } A \text { and } C \text { in } R \text {-modules }\right\}
$$

If there is no such $n$, then gl. $\operatorname{dim} \mathscr{P}=\infty$.

Proposition 2.5 ([2]). If $R$ is a hereditary ring, then $\operatorname{gl} \cdot \operatorname{dim} \underline{k}(\mathscr{F}) \leq 1$ for every coinjectively generated class $\underline{k}(\mathscr{F})$.

\section{THE SMALLEST PROPER CLASS CONTAINING W $\mathscr{S}$}

Let $\mathscr{S}$ be the class of all short exact sequences (which are called $\kappa$-elements by Zöschinger [12, p. 1]) $E: 0 \longrightarrow A \stackrel{f}{\longrightarrow} B \stackrel{g}{\longrightarrow} C \longrightarrow 0$ such that $\operatorname{Im} f$ has a supplement in $B$, that is, a minimal element in the set $\{V \subset B \mid V+\operatorname{Im} f=B\}$. We 
denote by $\mathscr{W} S$ the class of short exact sequences $E$, where $\operatorname{Im} f$ has (is) a weak supplement in $\mathrm{B}$, that is, there is a submodule $K$ of $B$ such that $\operatorname{Im} f+K=B$ and $\operatorname{Im} f \cap K \ll B$ and by $\mathscr{S}$ mall the class of short exact sequences $E$ where $\operatorname{Im} f \ll B$.

If $X$ is a $\mathscr{S}$ mall-submodule of an $R$-module $Y$, then $Y$ is a supplement of $X$ in $Y$, so $X$ is an $\mathscr{S}$-submodule of $Y$. If $U$ is an $\mathscr{S}$-submodule of an $R$-module $Z$, then a supplement $V$ of $U$ in $Z$ is also a weak supplement, therefore $U$ is a $\mathscr{W} \mathscr{S}$-submodule of $Z$. These arguments give the relation $\mathscr{S}$ mall $\subseteq \mathscr{S} \subseteq \mathscr{W} \mathscr{S}$ for any ring $R$. Our next example shows that neither of the classes $\mathscr{S}$ mall, $\mathscr{S}$ or $\mathscr{W} \mathscr{S}$ need to be a proper class in general. Therefore, it makes sense to study the smallest proper classes generated by them. Our main goal in this section will be to show that, for hereditary rings, these three classes generate the same proper class.

Example 3.1. Let $R=\mathbb{Z}$ and consider the composition $\beta \circ \alpha$ of the monomorphisms $\alpha: 2 \mathbb{Z} \longrightarrow \mathbb{Z}$ and $\beta: \mathbb{Z} \longrightarrow \mathbb{Q}$ where $\alpha$ and $\beta$ are the corresponding inclusions. Then the short exact sequence $0 \longrightarrow 2 \mathbb{Z} \stackrel{\beta \circ \alpha}{\longrightarrow} \mathbb{Q} \longrightarrow$ $\mathbb{Q} / 2 \mathbb{Z} \longrightarrow 0$ is in $\mathscr{S}$ mall and therefore in $\mathscr{W} \mathscr{S}$, but the short exact sequence $0 \longrightarrow 2 \mathbb{Z} \stackrel{\alpha}{\longrightarrow} \mathbb{Z} \longrightarrow \mathbb{Z} / 2 \mathbb{Z} \longrightarrow 0$ is not in $\mathscr{W} \mathscr{S}$ as $\operatorname{Rad} \mathbb{Z}=0$.

This example shows that $\operatorname{Ext}_{\text {Wy }}(\cdot, \cdot)$ need not be a subfunctor of $\operatorname{Ext}(\cdot, \cdot)$ since the elements from $\mathscr{W} S$ are not preserved with respect to the first variable. We begin by extending the class $\mathscr{W} \mathscr{S}$ to the class $\overline{\mathscr{W} S}$, which consists of all images of $\mathscr{W} \mathscr{S}$ elements of $\operatorname{Ext}\left(C^{\prime}, A\right)$ under $f^{*}: \operatorname{Ext}\left(C^{\prime}, A\right) \longrightarrow \operatorname{Ext}(C, A)$ for all homomorphisms $f: C \rightarrow C^{\prime}$. We will prove that $\overline{\mathscr{W} S}$ is the smallest proper class containing $\mathscr{W} \mathscr{S}$, that is, $<\mathscr{W} S>=\overline{\mathscr{W S}}$.

Definition 3.2. A short exact sequence $E: 0 \longrightarrow A \longrightarrow B \longrightarrow C \longrightarrow 0$ is said to be extended weak supplement if there is a short exact sequence $E^{\prime}: 0 \longrightarrow A \stackrel{f}{\longrightarrow}$ $B^{\prime} \longrightarrow C^{\prime} \longrightarrow 0$ such that $\operatorname{Im} f$ has (is) a weak supplement in $B$ and there is a homomorphism $g: C \rightarrow C^{\prime}$ such that $E=g^{*}\left(E^{\prime}\right)$, that is, there is a commutative diagram as follows:

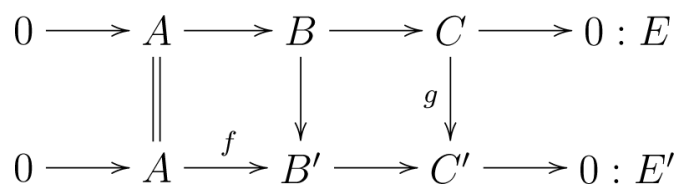

The class of all extended weak supplement short exact sequences will be denoted by $\overline{\mathscr{W} \mathscr{S}}$. So $\operatorname{Ext}_{\overline{\mathscr{W} S}}(C, A)=\left\{E: 0 \longrightarrow A \longrightarrow B \longrightarrow C \longrightarrow 0 \mid E=g^{*}\left(E^{\prime}\right)\right.$ for some $E^{\prime}$ : $0 \longrightarrow A \longrightarrow B \longrightarrow C^{\prime} \longrightarrow 0 \in \mathscr{W} S$ and $\left.g: C \rightarrow C^{\prime}\right\}$.

Lemma 3.3. For every homomorphism $f: A \rightarrow A^{\prime}$, the homomorphism $f_{*}$ : $\operatorname{Ext}(C, A) \longrightarrow \operatorname{Ext}\left(C, A^{\prime}\right)$ preserves short exact sequences from $\mathscr{W} S$.

Proof. Let $E: 0 \longrightarrow A \longrightarrow B \longrightarrow C \longrightarrow 0$ be a short exact sequence in the class WWS and $f: A \rightarrow A^{\prime}$ be an arbitrary homomorphism. We have the following diagram 
with exact rows:

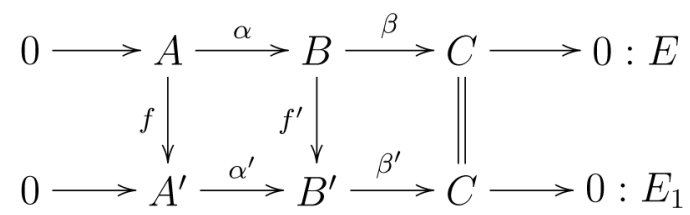

where $E_{1}=f_{*}(E)$.

If $V$ is a weak supplement of $\operatorname{Im} \alpha$ in $B$, then $\operatorname{Im} \alpha+V=B$ and $\operatorname{Im} \alpha \cap$ $V \ll B$. Then $f^{\prime}(V)+\operatorname{Im} \alpha^{\prime}=B^{\prime}$ by pushout diagram. We will show that $f^{\prime}(V) \cap$ $\operatorname{Im} \alpha^{\prime}=f^{\prime}(\operatorname{Im} \alpha \cap V)$. Since $f^{\prime}(\operatorname{Im} \alpha)=\alpha^{\prime}(\operatorname{Im} f)$, we obtain $f^{\prime}(V \cap \operatorname{Im} \alpha) \subseteq f^{\prime}(V) \cap$ $\operatorname{Im} \alpha^{\prime}$. For the other inclusion, let $x \in f^{\prime}(V) \cap \operatorname{Im} \alpha^{\prime}$. Then $x=f^{\prime}(v)=\alpha^{\prime}\left(a^{\prime}\right)$ for some $v \in V, a^{\prime} \in A^{\prime}$. We have $\beta(v)=\left(\beta^{\prime} \circ f^{\prime}\right)(v)=\beta^{\prime}(x)=\left(\beta^{\prime} \circ \alpha^{\prime}\right)\left(a^{\prime}\right)=0$, so $v \in$ $V \cap \operatorname{Ker} \beta=V \cap \operatorname{Im} \alpha$. Therefore $x=f^{\prime}(v) \in f^{\prime}(V \cap \operatorname{Im} \alpha)$. Hence $f^{\prime}(V) \cap \operatorname{Im} \alpha^{\prime}=$ $f^{\prime}(\operatorname{Im} \alpha \cap V) \ll f^{\prime}(B) \subseteq B^{\prime}$. So $E_{1} \in \mathscr{W} \mathscr{S}$.

Lemma 3.4. For every homomorphism $f: A \rightarrow A^{\prime}$, the homomorphism $f_{*}$ : $\operatorname{Ext}(C, A) \longrightarrow \operatorname{Ext}\left(C, A^{\prime}\right)$ preserves short exact sequences from $\overline{\mathscr{W} S}$.

Proof. Let $E: 0 \longrightarrow A \longrightarrow B \longrightarrow C \longrightarrow 0$ be a short exact sequence in the class $\overline{\mathscr{W} S}$ and $f: A \rightarrow A^{\prime}$ be an arbitrary homomorphism. Then $E=g^{*}\left(E^{\prime}\right)$ for some $E^{\prime}: 0 \longrightarrow A \longrightarrow B_{1} \longrightarrow C_{1} \longrightarrow 0 \in \mathscr{W} S$ and a homomorphism $g: C \rightarrow C_{1}$. Therefore $E_{1}=f_{*}(E)=f_{*} \circ g^{*}\left(E^{\prime}\right)=g^{*} \circ f_{*}\left(E^{\prime}\right)=g^{*}\left(E_{1}^{\prime}\right)$, where $E_{1}^{\prime}=f_{*}\left(E^{\prime}\right) \in$ WWS by Lemma 3.3, and so $g^{*}\left(E_{1}^{\prime}\right)=E_{1} \in \overline{\mathscr{W} S}$.

Lemma 3.5. For every homomorphism $g: C^{\prime} \rightarrow C$, the homomorphism $g^{*}$ : $\operatorname{Ext}(C, A) \longrightarrow \operatorname{Ext}\left(C^{\prime}, A\right)$ preserves short exact sequences from $\overline{\mathscr{W} S}$.

Proof. Let $E: 0 \longrightarrow A \longrightarrow B \longrightarrow C \longrightarrow 0$ be a short exact sequence in the class $\overline{\mathscr{W} S}$ and $g: C^{\prime} \rightarrow C$ be an arbitrary homomorphism. Then $E=f^{*}\left(E_{1}\right)$ for some $E_{1} \in \mathscr{W} S$ and a homomorphism $f: C \rightarrow C_{1}$. Therefore $E^{\prime}=g^{*} \circ f^{*}\left(E_{1}\right)=(f \circ$ $g)^{*}\left(E_{1}\right)$. Since $E_{1} \in \mathscr{W} \mathscr{S}$, we have $E^{\prime} \in \overline{\mathscr{W} S}$.

Corollary 3.6. If the composition $f \circ g$ of homomorphisms $g$ and $f$ is a $\overline{\mathscr{W S}}$ monomorphism, then $g$ is a $\overline{\mathscr{W S}}$-monomorphism.

Proposition 3.7. If $E_{1}, E_{2} \in \operatorname{Ext}_{\text {WS }}(C, A)$, then $E_{1} \oplus E_{2} \in \operatorname{Ext}_{\text {WS }}(C \oplus C, A \oplus A)$.

Proof. Let $E_{1}, E_{2} \in \operatorname{Ext}_{\mathscr{W} S}(C, A)$, then there exists a submodule $V_{i}$ in $B_{i}$ such that $V_{i}+A=B_{i}$ and $V_{i} \cap A \ll B_{i}$ where $i=1,2$. Hence

$$
E_{1} \oplus E_{2}: 0 \longrightarrow A \oplus A \longrightarrow B_{1} \oplus B_{2} \longrightarrow C \oplus C \longrightarrow 0 \in \mathscr{W} \mathscr{S}
$$

since $(A \oplus A)+\left(V_{1} \oplus V_{2}\right)=B_{1} \oplus B_{2} \quad$ and $(A \oplus A) \cap\left(V_{1} \oplus V_{2}\right)=\left(V_{1} \cap A\right) \oplus\left(V_{2} \cap\right.$ $A) \ll B_{1} \oplus B_{2}$.

Corollary 3.8. $\operatorname{Ext}_{\overline{\mathscr{W} Y}}(C, A)$ is a subgroup of $\operatorname{Ext}(C, A)$ for every modules $C$ and $A$. 
Proof. Let $E_{1}, E_{2} \in \operatorname{Ext}_{\overline{W W S}}(C, A)$. We have the following commutative diagram with exact rows:

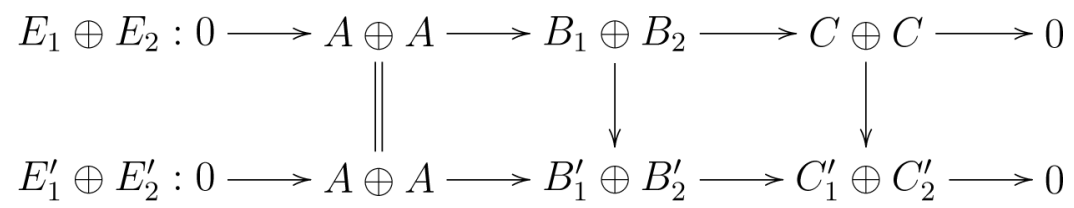

where $E_{1}$ and $E_{2}$ are the images of short exact sequences $E_{1}^{\prime}$ and $E_{2}^{\prime}$ from WWS respectively. $E_{1}^{\prime} \oplus E_{2}^{\prime}$ is $\mathscr{W} \mathscr{S}$-element by Proposition 3.7 and so $E_{1} \oplus E_{2}$ is $\overline{\mathscr{W} S}$ element. Since $E_{1}+E_{2}=\nabla_{A}\left(E_{1} \oplus E_{2}\right) \Delta_{C}$ where $\Delta_{C}: c \mapsto(c, c)$ is the diagonal map and $\nabla_{A}:\left(a_{1}, a_{2}\right) \mapsto a_{1}+a_{2}$ is the codiagonal map, $E_{1}+E_{2}$ is in $\overline{\mathscr{W S}}$ by Lemma 3.4 and Lemma 3.5 .

Now by Theorem 1.1 in [9], to prove that $\overline{\mathscr{W S}}$ is a proper class, it remains only to show that the composition of two $\overline{\mathscr{W S}}$-monomorphisms is a $\overline{\mathscr{W S}}$ monomorphism. First, we prove some useful results.

Lemma 3.9. The composition $f \circ g$ of a splitting monomorphism $g: A \rightarrow B$ and $a$ WS-monomorphism $f: B \rightarrow C$ is a $\overline{\mathscr{W} S}$-monomorphism.

Proof. Without loss of generality we can assume that $A$ is a direct summand in $B$, $B \leq C$ and $g$ and $f$ are inclusion maps. Let $B=A \oplus B^{\prime}$. Clearly $f=\nabla_{C} \circ\left(\left.1_{C} \oplus f\right|_{B^{\prime}}\right) \circ$ $\left(\left.\left.f\right|_{A} \oplus 1\right|_{B^{\prime}}\right)$. Since $f$ is a $\mathscr{W} \mathscr{S}$-monomorphism $\left.\left.f\right|_{A} \oplus 1\right|_{B^{\prime}}$ is a $\overline{\mathscr{W} \mathscr{S}}$-monomorphism by Corollary 3.6. We have the following commutative diagram with exact rows:

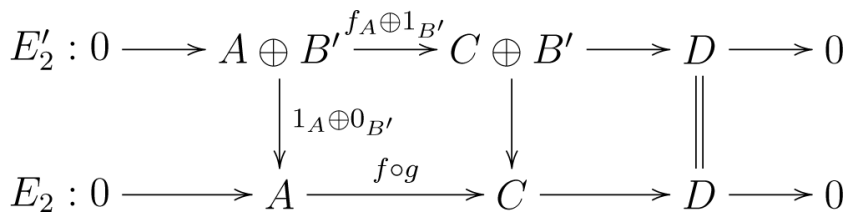

where $D=C / A$. So $f \circ g$ is a $\overline{\mathscr{W S}}$-monomorphism by Lemma 3.4.

Lemma 3.10. The composition of a S mall-epimorphism $f$ and a WS-epimorphism $g$ is a WOS-epimorphism.

Proof. Without loss of generality we can assume that $f: B \rightarrow B / K$ is a canonical epimorphism, $K \leq A \leq B, K \ll B$ and $g: B / K \rightarrow B / A$ is defined as in Third Isomorphism Theorem, so $g \circ f: B \rightarrow B / A$ is the canonical epimorphism. Then $A / K$ has a weak supplement $D / K$ in $B / K$, that is, $D / K+A / K=B / K$ and $(D \cap A) / K \ll$ $B / K$. Therefore $A+D=B$ and $A \cap D \ll B$ by Proposition 5.17 in [4], that is, $D$ is a weak supplement of $A$ in $B$.

Lemma 3.11. If the ring $R$ is hereditary, then the composition of a Smallepimorphism and a $\overline{\mathscr{W S}}$-epimorphism is a $\overline{\mathscr{W} S}$-epimorphism.

Proof. Let $f: B \rightarrow B^{\prime}$ be a small epimorphism and $h: B^{\prime} \rightarrow C$ be a $\overline{\mathscr{W} S}$ epimorphism, $K=\operatorname{Ker} f, A=\operatorname{Ker}(h \circ f), A^{\prime}=\operatorname{Ker} h$. Since $h$ is a $\overline{\mathscr{W} \mathscr{S}}$-epimorphism, there is a commutative diagram with exact rows and with $E_{3} \in \mathscr{W} S$ : 


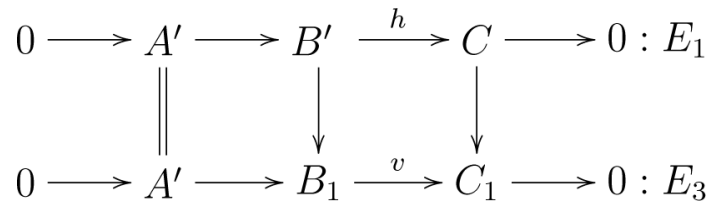

Since $g=\left.f\right|_{A}: A \rightarrow A^{\prime}$ is an epimorphism we have an exact sequence

$$
\cdots \longrightarrow \operatorname{Ext}^{1}\left(C_{1}, N\right) \longrightarrow \operatorname{Ext}^{1}\left(C_{1}, A\right) \stackrel{g_{*}}{\longrightarrow} \operatorname{Ext}^{1}\left(C_{1}, A^{\prime}\right) \longrightarrow \operatorname{Ext}^{2}\left(C_{1}, N\right) \longrightarrow \cdots
$$

where $N=\operatorname{Ker} g$. Since $R$ is hereditary $\operatorname{Ext}^{2}\left(C_{1}, N\right)=0$, therefore $g_{*}$ is an epimorphism, that is $E_{3}=\operatorname{Ext}^{1}\left(1_{C_{1}}, g\right)\left(E_{4}\right)$ for some $E_{4}: 0 \longrightarrow A \longrightarrow B_{2} \longrightarrow$ $C_{1} \longrightarrow 0$. Then we have the following commutative exact diagram:

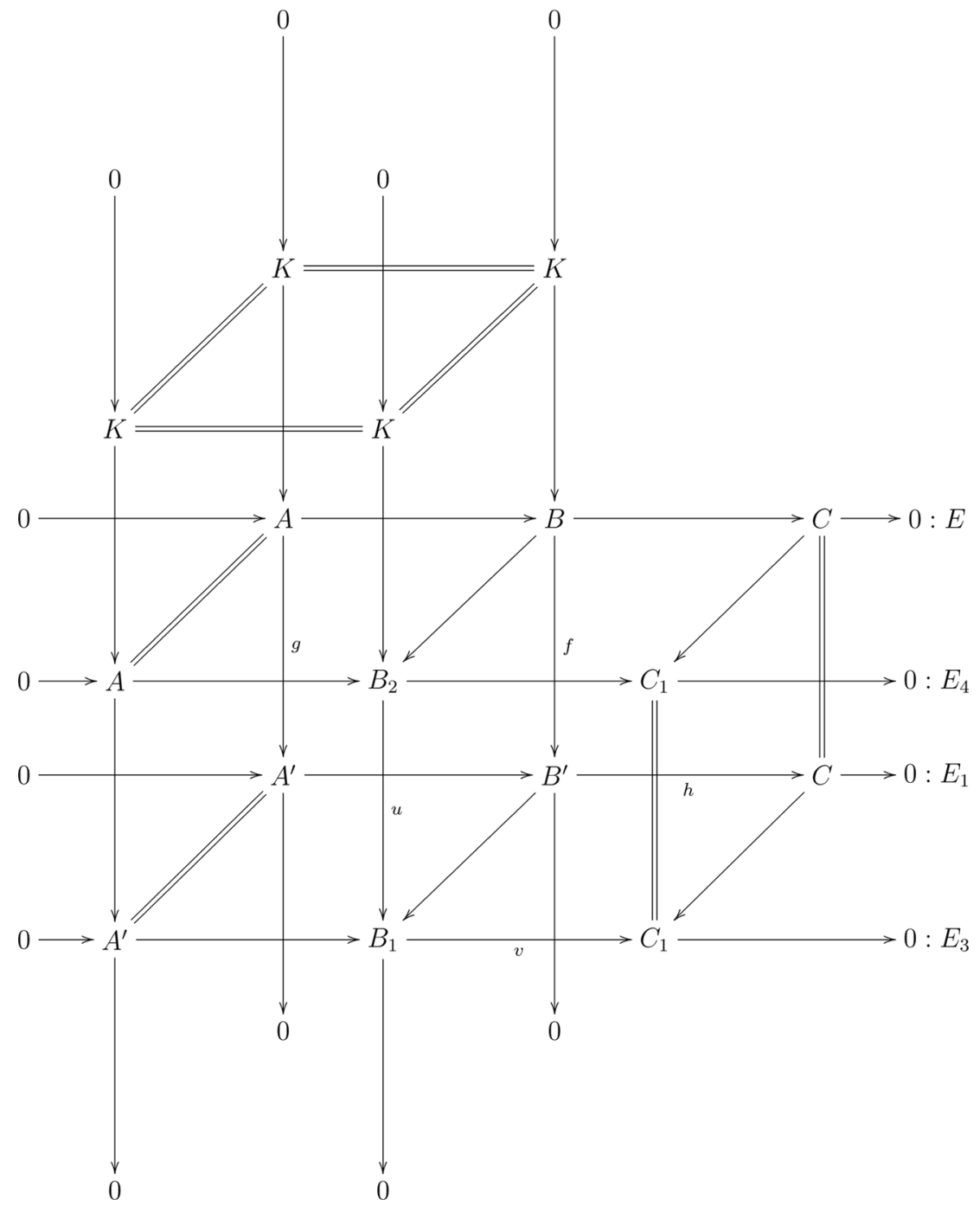


Since $K=\operatorname{Ker} f \ll B, u: B_{2} \rightarrow B_{1}$ is a 9 mall epimorphism. Therefore, $v \circ u$ is a WSS-epimorphism by Lemma 3.10 , that is, $E_{4} \in \mathscr{W} \mathscr{S}$. Then $E \in \overline{\mathscr{W} S}$.

Theorem 3.12. If $R$ is a hereditary ring, $\overline{\mathscr{W} S}$ is a proper class.

Proof. By Lemma 3.4, Lemma 3.5, Corollary 3.8, we have $\operatorname{Ext}_{\mathscr{W W Y}}^{1}(C, A)$ is a subfunctor of $\operatorname{Ext}_{R}^{1}(C, A)$ and $\operatorname{Ext}_{\mathscr{W} \mathscr{S}}^{1}(C, A)$ is a subgroup of $\operatorname{Ext}_{R}^{1}(C, A)$ for every $R$-modules $A, C$. By Theorem 1.1 in [9], it is sufficient to show that the composition of two $\overline{\mathscr{W S}}$-monomorphisms is a $\overline{\mathscr{W S}}$-monomorphism. Let $f: A \rightarrow B$ and $g: B \rightarrow$ $C$ be a $\overline{\mathscr{W S}}$-monomorphisms. Then for the short exact sequence $E_{2}: 0 \longrightarrow B \stackrel{g}{\longrightarrow}$ $C \longrightarrow F \longrightarrow 0$ from $\overline{\mathscr{W} Y S}$ we have $E_{2}=h^{*}\left(E_{2}^{\prime}\right)$ for some $E_{2}^{\prime}: 0 \longrightarrow B \longrightarrow C^{\prime} \longrightarrow$ $F^{\prime} \longrightarrow 0 \in \mathscr{W S}$ and homomorphism $h: F \rightarrow F^{\prime}$. Therefore, we have a commutative diagram with exact rows and columns:

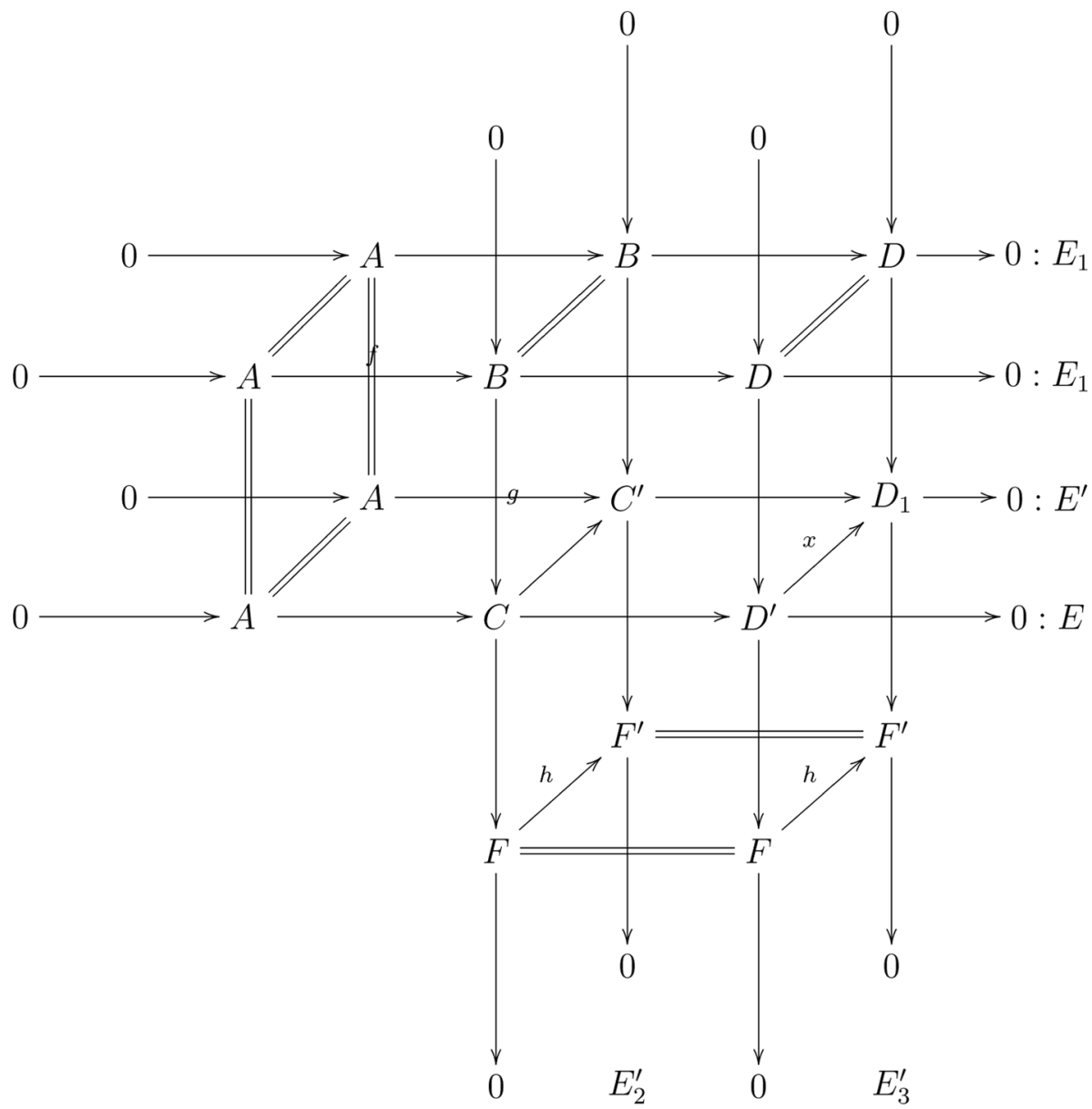

$E_{2} \quad E_{3}$ 
where $E_{2}$ and $E_{3}$ are images of $E_{2}^{\prime}$ and $E_{3}^{\prime}$, respectively, under the first variable. Now for the short exact sequence $E_{1}: 0 \longrightarrow A \stackrel{f}{\longrightarrow} B \longrightarrow D \longrightarrow 0 \in \overline{\mathscr{W} S}$, we have $E_{1}=u^{*}\left(E_{1}^{\prime}\right)$ for some $E_{1}^{\prime}: 0 \longrightarrow A \longrightarrow B_{1} \longrightarrow D_{2} \longrightarrow 0 \in \mathscr{W} S$ and homomorphism $u: D \rightarrow D_{2}$. Therefore, we have a commutative diagram with exact rows and columns:

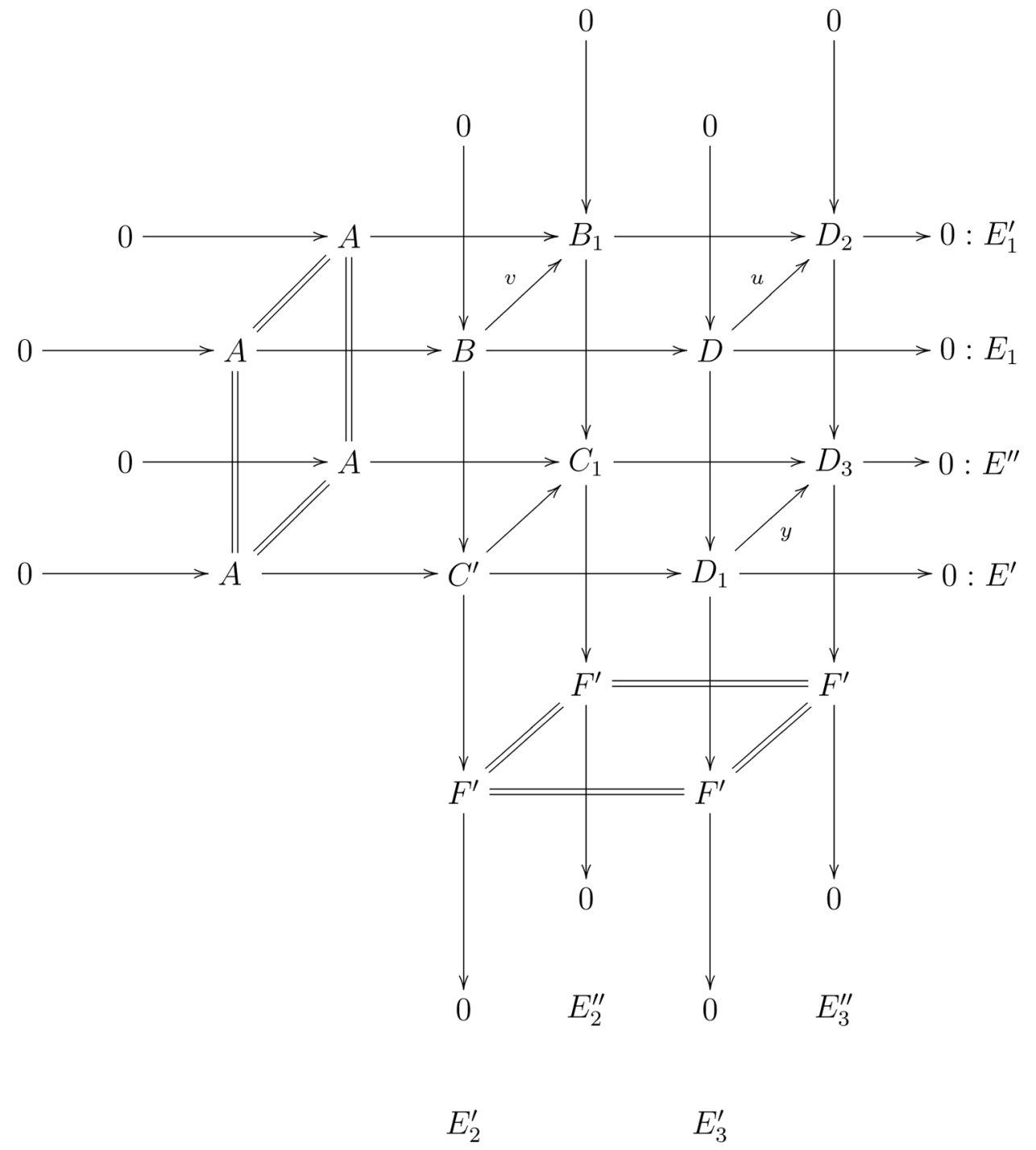

where $E_{2}^{\prime \prime}=v_{*}\left(E_{2}^{\prime}\right), E_{3}^{\prime \prime}=u_{*}\left(E_{3}^{\prime}\right)$. Without loss of generality, we can assume that $A \leq B_{1} \leq C_{1}$. Since $E_{1}^{\prime} \in \mathscr{W} \mathscr{S}$, there is a submodule $K$ of $B_{1}$ such that $A+K=B_{1}$ and $A \cap K \ll B_{1}$. Then $A /(A \cap K) \oplus K /(A \cap K)=B_{1} / A \cap K$, that is, $A /(A \cap K)$ is a direct summand in $B_{1} /(A \cap K)$. Then we have the following diagram with exact rows and columns: 


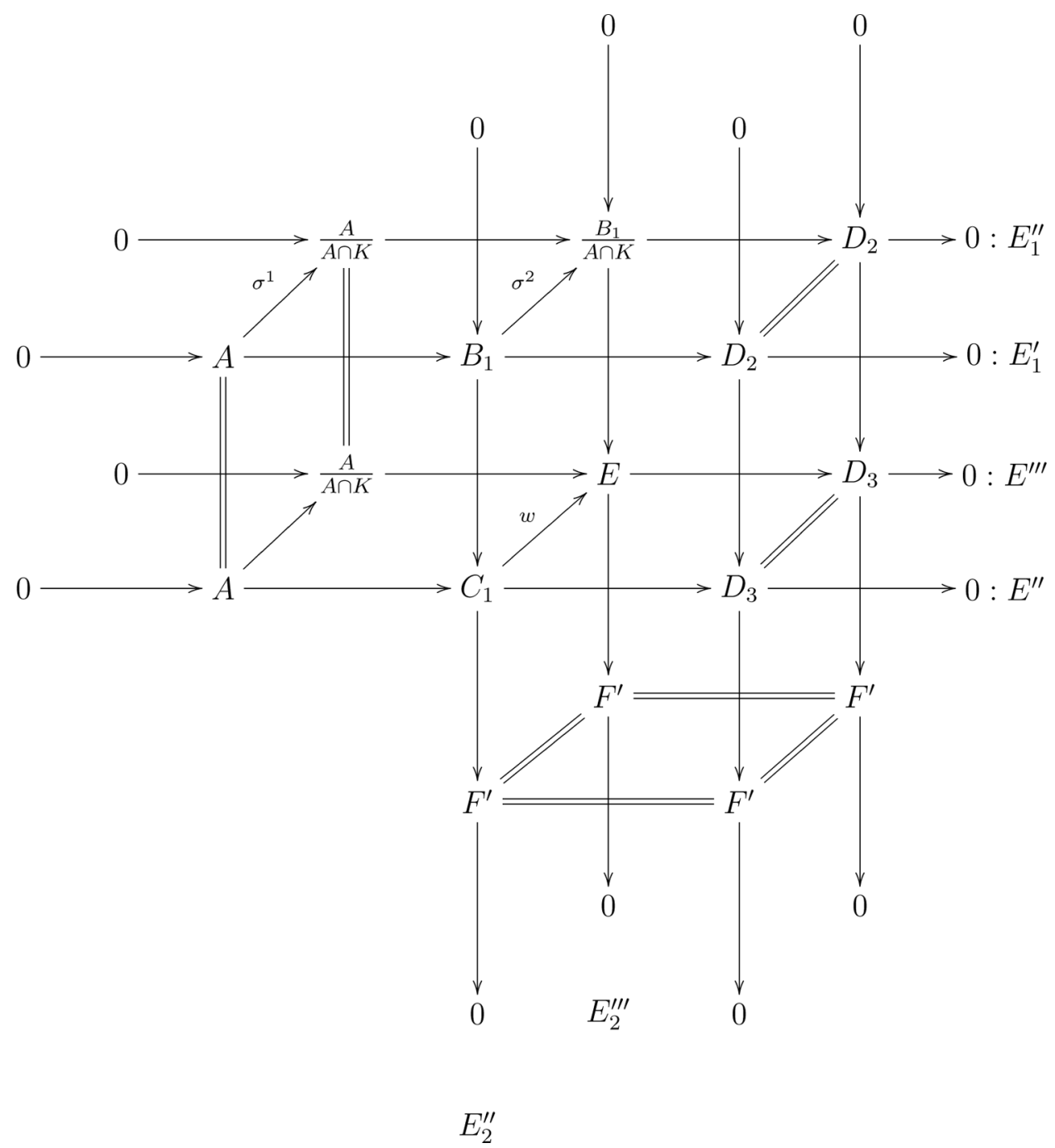

where $\sigma^{1}: A \rightarrow A /(A \cap K)$ and $\sigma^{2}: B_{1} \rightarrow B_{1} /(A \cap K)$ are canonical epimorphisms, $E_{1}^{\prime \prime}=\sigma_{*}^{1}\left(E_{1}^{\prime}\right), E_{2}^{\prime \prime \prime}=\sigma_{*}^{2}\left(E_{2}^{\prime \prime}\right)$. Since $E_{2}^{\prime} \in \mathscr{W} \mathscr{S}, E_{2}^{\prime \prime}$ and $E_{2}^{\prime \prime \prime}$ are in $\mathscr{W} \mathscr{S}$. By Lemma 3.9, $E^{\prime \prime \prime} \in \mathscr{W S}$. By $3 \times 3$ Lemma Ker $w=\operatorname{Ker} \sigma^{2}=A \cap K \ll C_{1}$. Therefore, by Lemma 3.11, we have $E^{\prime \prime} \in \overline{\mathscr{W} S}$. Now $E=(y \circ x)^{*}\left(E^{\prime \prime}\right) \in \overline{\mathscr{W} S}$ by Lemma 3.5.

Corollary 3.13. If $R$ is hereditary, then $\langle\mathscr{S}$ mall $\rangle=\langle\mathscr{S}\rangle=\langle\mathscr{W} \mathscr{S}\rangle=\overline{\mathscr{W} \mathscr{S}}$.

Proof. The equality $\langle\mathscr{S}$ mall $\rangle=\langle\mathscr{S}\rangle=\langle\mathscr{W} \mathscr{S}\rangle$ had been proved in [5]. Since $\langle\mathscr{W} \mathscr{S}\rangle$ is the smallest proper class containing $\mathscr{W} S$ and $\mathscr{W} S$ is contained in the proper class $\overline{\mathscr{W S}},\langle\mathscr{W} \mathscr{S}\rangle \subseteq \overline{\mathscr{W S}}$. Conversely, let $E \in \overline{\mathscr{W S}}$. Then $E=f^{*}\left(E^{\prime}\right)$ for some $E^{\prime} \in$ WWS and some homomorphism $f$. Therefore, $E \in<\mathscr{W} \mathscr{S}>$ since $\operatorname{Ext}_{<\mathscr{W} S>>}^{1}(\cdot, \cdot)$ is a subfunctor of $\operatorname{Ext}^{1}(\cdot, \cdot)$. 


\section{HOMOLOGICAL OBJECTS OF $\overline{\mathscr{W S}}$}

We have shown in the above section that, for hereditary rings $R$, the classes $\mathscr{S}$ mall, $\mathscr{S}$ and $\mathscr{W} \mathscr{S}$ generate the same proper class $\langle\mathscr{S}$ mall $\rangle$. Therefore, our interest in this section will focus on the study of the main properties of this proper class, that is, injective, projective, coprojective or coinjective modules with respect to the short exact sequences living in $\overline{\mathscr{W} S}$.

We begin by studying coinjective modules with respect to $\overline{\mathscr{W S}}$. This will allow us to prove that $\overline{\mathscr{W} S}$ has global dimension bounded by 1 . Along this section, $R$ will denote a hereditary ring unless otherwise stated.

Theorem 4.1. For an R-module A, the following statements are equivalent:

(i) $A$ is $\overline{\mathscr{W S}}$-coinjective;

(ii) There is a submodule $N$ of $A$ such that $N$ is small in the injective hull $\widehat{A}$ of $A$ and $A / N$ is injective;

(iii) A has a weak supplement in its injective hull $\widehat{A}$;

(iv) A has a weak supplement in some injective module I.

Proof. $(i \Rightarrow i i)$ Let $f: A \rightarrow \widehat{A}$ be an injective hull of $A$. Since $A$ is $\overline{\mathscr{W} \mathscr{S}}$-coinjective, the short exact sequence $E: 0 \longrightarrow A \stackrel{f}{\longrightarrow} \widehat{A} \longrightarrow C \longrightarrow 0 \in \overline{\mathscr{W} S}$, that is, there is a commutative diagram

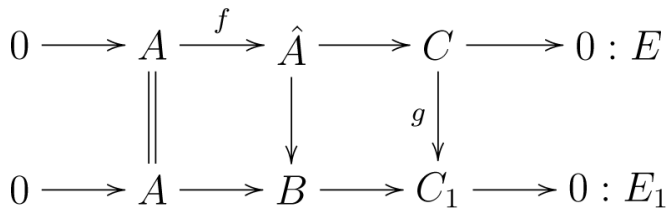

with exact rows and with $E_{1} \in \mathscr{W} \mathscr{S}$. Since $\operatorname{Im} f \unlhd \widehat{A}, g$ is a monomorphism. Without loss of generality, we can assume that $A \leq \widehat{A} \leq B$ and $f, g$ are inclusion maps. Then there is a submodule $V$ of $B$ such that $A+V=B$ and $N=A \cap V \ll B$. Since $\widehat{A}$ is injective, it is a direct summand of $B$, and therefore, $N \ll \widehat{A}$. Now $A / N$ is a direct summand in $B / N$ which is injective.

(ii $\Rightarrow$ iii) $\quad$ By the hypothesis, we obtain the following diagram where $N$ is small in $\widehat{A}$ and $A^{\prime}$ is injective:

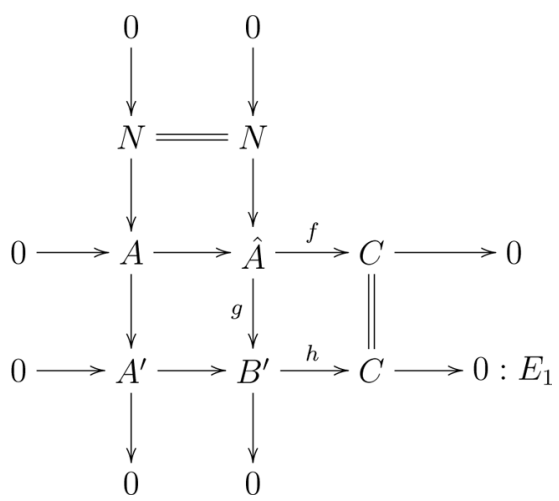


Then $g$ is a Small-epimorphism and $h$ is a $\mathscr{S}$ plit-epimorphism. So $f=h \circ g$ is $\mathscr{W} \mathscr{S}$ epimorphism by Lemma 3.10 .

$$
\begin{aligned}
& (i i i \Rightarrow i v) \quad \text { Clear. } \\
& (i v \Rightarrow i) \quad \text { By (1.7) in [8], since } \mathscr{W} \mathscr{S} \subseteq \overline{\mathscr{W} S} .
\end{aligned}
$$

Definition 4.2. A module $M$ is called radical-supplemented, if $\operatorname{Rad}(M)$ has a supplement in $M$.

Since every $\overline{\mathscr{W} S}$-coinjective module has a weak supplement in its injective hull by the theorem, Folgerung 1 in [14] gives the following description of $\overline{\mathscr{W S}}$ coinjective modules over a Dedekind domain.

Corollary 4.3. If $R$ is a Dedekind domain and $M$ is $\overline{\mathscr{W S}}$-coinjective, then $T(M)$ is radical-supplemented and there exists $n \geq 0$ with $\mathfrak{p}-\operatorname{Rank}(M / T(M)) \leq n$ for all maximal ideals $\mathfrak{p}$.

By (1.9) and the remark after (1.15) in [8] and also by Corollary of Lemma 2.1 in [14] we obtain the following corollary.

Corollary 4.4. The class of $\overline{\mathscr{W S}}$-coinjective $R$-modules is closed under extensions and factor modules. This class contains torsion-free modules with finite rank.

Definition 4.5. A module $M$ is said to be coatomic if $\operatorname{Rad}(M / U) \neq M / U$ for every proper submodule $U$ of $M$, or equivalently, every proper submodule of $M$ is contained in a maximal submodule of $M$.

Lemma 2.1 in [14] characterizes modules $M$ that have a weak supplement in the injective hull $\widehat{M}$. It gives the following characterization of $\overline{\mathscr{W} S}$-coinjective modules.

Corollary 4.6. Let $R$ be a Dedekind domain. An R-module $M$ is $\overline{\mathscr{W S}}$-coinjective if and only if $M$ has a dense coatomic submodule.

The following proposition follows from the proof of Lemma 3.3 in [12].

Proposition 4.7. Let $R$ be a Dedekind domain, $A, B$ be $R$-modules, and $A \subseteq B$. Then $A \ll B$ if and only if $A$ is coatomic and $A \subseteq \operatorname{Rad} B$.

Corollary 4.8. Over a Dedekind domain every coatomic module is $\overline{\mathscr{W} S}$-coinjective.

Proof. Since $\operatorname{Rad} I=I$ for every injective module $I$ every coatomic submodule is small in its injective hull by Proposition 4.7 and therefore is $\overline{\mathscr{W} \mathscr{S}}$-coinjective by Theorem 4.1.

$\overline{\mathscr{W S}}$-coinjective modules need not be either injective or coatomic. For example the $\mathbb{Z}$-module $\mathbb{Q}$ is a weakly supplemented module and every submodule of $\mathbb{Q}$ is $\overline{\mathscr{W S}}$-coinjective. If we assume that every proper submodule of $\mathbb{Q}$ is coatomic, then 
we come to the conclusion that $\mathbb{Q}$ is hollow. But $\mathbb{Q}$ is not hollow and so $\mathbb{Q}$ has a $\overline{\mathscr{W S}}$-coinjective proper submodule which is not coatomic. And also the group of $p$-adic numbers, $J_{p}$, is $\overline{\mathscr{W} S}$-coinjective but not coatomic.

Recall that a module $M$ over a commutative ring $R$ is bounded if $r M=0$ for some $0 \neq r \in R$.

Proposition 4.9. If $R$ is a Dedekind domain every bounded R-module is $\overline{\mathscr{W S S}}$ coinjective.

Proof. Let $B$ be a bounded $R$-module and $I$ be an injective hull of $B$. We will show that $B \ll I$. Suppose $B+X=I$ for some $X \subset I$. Since $B$ is bounded, there exists $0 \neq r \in R$ such that $r B=0$. Then $I=r I=r B+r X=r X$, since $I$ is divisible. Therefore $X=I$ and so $B \ll I$. Since $I$ is injective, it is $\langle\mathscr{S}$ mall $\rangle$-coinjective. Then $B$ is $\langle\mathscr{S}$ mall $\rangle$-coinjective by (1.8) in [8].

Lemma 4.10 ([5, Lemma 4.5]). Let $S$ be a discrete valuation ring, $A$ be a reduced torsion $S$-module, and $B$ be a bounded submodule of $A$. If $A / B$ is divisible, then $A$ is also bounded.

Lemma 4.11. Let $M$ be a torsion and reduced module over a discrete valuation ring. Then $M$ is $\overline{\mathscr{W} S}$-coinjective if and only if $M$ is coatomic.

Proof. Let $M$ be $\overline{\mathscr{W S}}$-coinjective. Then $M$ has a dense coatomic submodule $N$ by Corollary 4.6. Since $M$ is torsion, $N$ is torsion. Since $N$ is coatomic, $N=B+R^{n}$ with $p^{m} B=0$ for some $n \in \mathbb{N}$ [11, Lemma 2.1(iii)]. Since $N$ is torsion $R^{n}=0$ and therefore $N$ is bounded. By Lemma 4.10, $M$ is bounded and so it is coatomic.

Now let $M$ be coatomic. Since any coatomic module is small in its injective hull, it is $\langle\mathscr{S}$ mall $\rangle$-coinjective and also it is $\overline{\mathscr{W} \mathscr{S}}$-coinjective.

Definition 4.12 (see [6]). A module $M$ is called small if $M$ can be embedded as a small submodule into some submodule $N$, or equivalently $M$ is small in its injective hull. class.

The following proposition shows that $\overline{\mathscr{W} Y}$ is a coinjectively generated proper

Proposition 4.13. $\overline{\mathscr{W} S}=\underline{k}\left(\mathscr{S}_{m}\right)$ where $\mathscr{S}$ is the class of all small modules.

Proof. By Theorem 4.1, all small modules are $\overline{\mathscr{W} S}$-coinjective, so $k(\mathscr{S} m) \subseteq \overline{\mathscr{W} S}$. On the other hand, clearly $\mathscr{S}$ mall $\subseteq \underline{k}\left(\mathscr{S}_{m}\right)$, and therefore, $\overline{\mathscr{W} \mathscr{S}}=\langle\mathscr{S}$ mall $\rangle \subseteq$ $\underline{k}(\mathscr{S} m)$.

From Proposition 4.7 and from the proof of Corollary 4.8, we see that over Dedekind domains, small modules coincide with coatomic modules, so we have the following corollary.

Corollary 4.14. Over a Dedekind domain $\overline{\mathscr{W} S}=\underline{k}(\operatorname{co} A)$ where $\operatorname{co} \mathscr{A}$ is the class of all coatomic modules. 
Now by Propositions 4.13 and 2.5 , we obtain the following evaluation for the global dimension of the class $\overline{\mathscr{W} S}$.

Theorem 4.15. gl.dim $\overline{\mathscr{W} \mathscr{S}} \leq 1$.

We do not know whether there are $\overline{\mathscr{W S}}$-injective modules which are not injective in general, but for Dedekind domains we have the following proposition.

Proposition 4.16. Over a Dedekind domain $R$, the only $\overline{\mathscr{W} S}$-injective modules are the injective $R$-modules.

Proof. Let $M$ be a $\overline{\mathscr{W S}}$-injective module and $I$ be an ideal of $R$ and $f: I \rightarrow M$ be a homomorphism. The ideal $I$ is finitely generated, therefore it is $\overline{\mathscr{W} S}$-coinjective by Corollary 4.8. Then the inclusion map $I \rightarrow R$ is a $\overline{\mathscr{W}}$-monomorphism, and therefore, $f$ can be extended to a homomorphism $g: R \rightarrow M$. It means that $M$ is injective.

We obtain the following Corollary from Proposition 4.16 by using Proposition 2.2.

Corollary 4.17. Over a Dedekind domain $R$, the only WSS-injective modules are the injective $R$-modules.

We finish this section with some results about $\overline{\mathscr{W} \mathscr{S}}$-projective and $\overline{\mathscr{W} \mathscr{S}}$ coprojective modules.

By Proposition 4.13 and by the dual statement of Proposition 1.2 in [10], $\overline{\mathscr{W} S}$ is generated by the short exact sequences of the form $0 \longrightarrow M \longrightarrow I \longrightarrow B \longrightarrow 0$ where $M$ is a small module and $I$ is injective. By Proposition 2.4 in [10], we have the following criteria for the $\overline{\mathscr{W} Y}$-projective modules.

Proposition 4.18. A module $C$ is $\overline{\mathscr{W S}}$-projective if and only if $\operatorname{Ext}_{R}(C, M)=0$ for every small module $M$.

Corollary 4.19. Over a Dedekind domain, a module $C$ is $\overline{\mathscr{W} S}$-projective if and only if $\operatorname{Ext}_{R}(C, M)=0$ for every coatomic module $M$.

Corollary 4.20. Every finitely presented module is $\overline{\mathscr{W} S}$-coprojective.

Proof. Let $M$ be a finitely presented module. Then there is a projective module $P$ and its finitely generated submodule $K$ such that $M \cong F / K$. Then $K$ is $\overline{\mathscr{W S}}$ coinjective by Corollary 4.14, therefore $M$ is $\overline{\mathscr{W} S}$-coprojective by (1.12) in [8].

\section{COATOMIC SUPPLEMENT SUBMODULES}

We finish this article by introducing the notion of coatomic supplement modules and showing that, for Dedekind domains, the class $\overline{\mathscr{W} Y}$ can also be constructed in terms of them. Throughout this section, rings will be hereditary, unless otherwise stated. 
Definition 5.1. Let $U$ be a submodule of an $R$-module $M$. We will say that $U$ is a coatomic supplement of a submodule $V$ of $M$ if there exists a submodule $V$ of $M$ such that $M=U+V$ and $U \cap V$ is coatomic.

Our main result in this section will show that, for Dedekind domains, the class CAS of short exact sequences $E: 0 \longrightarrow A \stackrel{f}{\longrightarrow} B \longrightarrow C \longrightarrow 0$ satisfying that $\operatorname{Im} f$ has a coatomic supplement in $B$ coincides with the class $\overline{\mathscr{W} Y S}$.

Lemma 5.2. If $g: C^{\prime} \rightarrow C$, then $g^{*}: \operatorname{Ext}(C, A) \rightarrow \operatorname{Ext}\left(C^{\prime}, A\right)$ preserves $\sigma$-elements.

Proof. Let $E: 0 \longrightarrow A \longrightarrow B \longrightarrow C \longrightarrow 0$ is a short exact sequence in $\operatorname{Ext}(C, A)$ and $g: C^{\prime} \rightarrow C$ be an arbitrary homomorphism. The following diagram is commutative with exact rows,

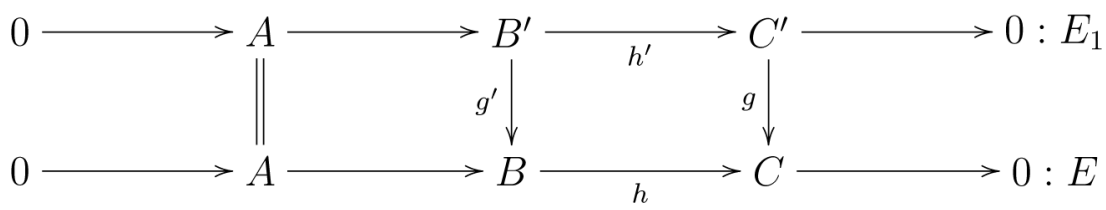

where $g^{*}(E)=E_{1}$.

Let $V$ be a coatomic supplement of $\operatorname{Ker} h$ in $B$, that is, $\operatorname{Ker} h+V=B$ and $V \cap$ Ker $h$ is coatomic. Then $g^{\prime-1}(V)+\operatorname{Ker} h^{\prime}=B^{\prime}$ by pullback diagram. Since $g^{\prime}$ induces an isomorphism between $D^{\prime}=g^{\prime-1}(V) \cap \operatorname{Ker} h^{\prime}$ and $D=V \cap \operatorname{Ker} h$ and epimorphic image of coatomic module coatomic, $D^{\prime}$ is coatomic.

Theorem 5.3. Over a Dedekind domain R, the class $\mathscr{C A S}$ coincides with the class $\overline{\mathscr{W} S}$.

Proof. Assume that $A$ has a coatomic supplement in $B$, then there exists a submodule $V$ of $B$ such that $B=A+V$ and $A \cap V$ is coatomic. So we have the following commutative diagram with exact columns and rows:

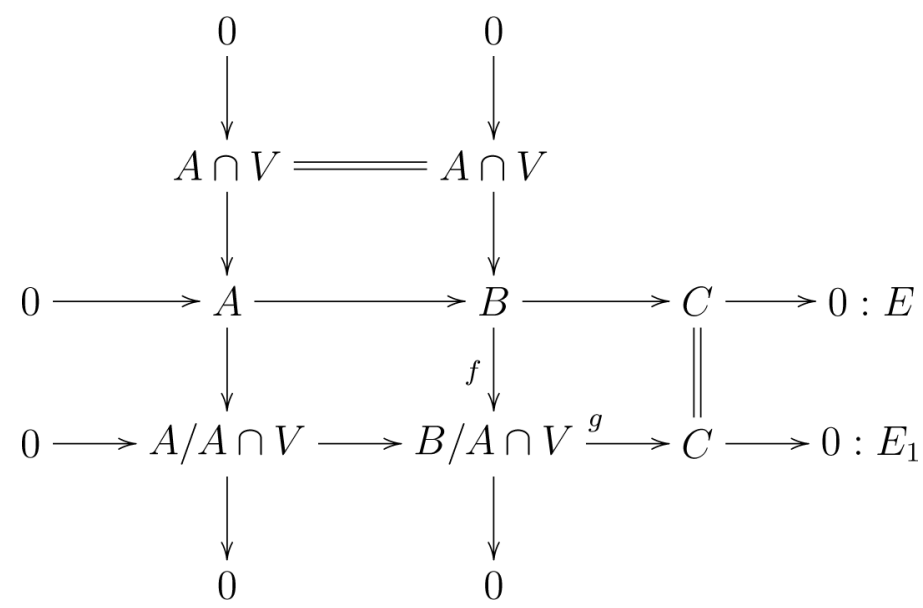


Clearly, $g$ is a $\mathscr{S}$ plit-epimorphism, and since coatomic module is $\overline{\mathscr{W} \mathscr{S}}$-coinjective, $f$ is a $\overline{\mathscr{W S}}$-epimorphism. Then, the composition $g \circ f$ is a $\overline{\mathscr{W S}}$-epimorphism. So, $E$ is in $\overline{\mathscr{W S}}$. To prove the converse, let $E \in \overline{\mathscr{W S}}$. Then there is $E_{1}$ in the class $\mathscr{W} \mathscr{S}$ such that there is the following commutative diagram with exact rows:

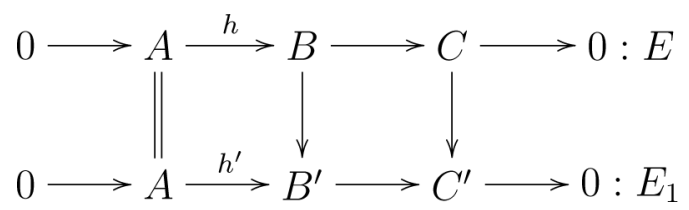

If $V$ is weak supplement of $\operatorname{Im} h^{\prime}$ in $B^{\prime}$, then $\operatorname{Im} h^{\prime}+V=B^{\prime}$ and $\operatorname{Im} h^{\prime} \cap V \ll B^{\prime}$ and so $\operatorname{Im} h^{\prime} \cap V$ is coatomic by Proposition 4.7. By Lemma 5.2, $E$ is in $\mathscr{C A S}$.

Let $R$ be a discrete valuation ring. If $A$ is a coatomic submodule of $B$, then since $B / \operatorname{Rad}(B)$ is semisimple,

$$
B / \operatorname{Rad}(B)=X / \operatorname{Rad}(B) \oplus(A+\operatorname{Rad}(B)) / \operatorname{Rad}(B)
$$

for some submodule $X$ of $B$. Therefore, $X+A=B$ and since $X \cap A \leq \operatorname{Rad}(B)$ and $X \cap A \leq \operatorname{Rad}(B)$ is coatomic, $X \cap A \ll B$. So, every coatomic module has a weak supplement in every extension. By Corollary $4.14 \overline{\mathscr{W} \mathscr{S}}$ is coinjectively generated by coatomic modules. Then by Theorem 2.3 and by Lemma 3.3 we have the following corollary.

Corollary 5.4. If $R$ is a discrete valuation ring, $\mathscr{C A S}=\overline{\mathscr{W} S}=\underline{k}(\mathscr{C A})=\mathscr{W} \mathscr{S}$, so WSS is a proper class.

\section{ACKNOWLEDGMENT}

The authors express their gratitude to the referee for a careful reading of the article and for suggesting several improvements.

Work supported by TÜBITAK Project-107T709.

\section{REFERENCES}

[1] Alizade, R. (1985a). On proper kepka classes. Math. Notes 37:268-273.

[2] Alizade, R. (1985b). Global dimension of some proper classes. Uspekhi Mat. Nauk $1: 181-182$.

[3] Anderson, I., Fuller, K. (1992). Rings and Categories of Modules. New York: SpringerVerlag.

[4] Clark, J., Lomp, J. C., Vanaja, N., Wisbauer, R. (2006). Lifting Modules: Supplements and Projectivity in Module Theory. Frontiers in Mathematics. Basel: Birkhäuser Verlag.

[5] Demirci, Y. M. (2009). Proper Class Generated By Submodules That Have Supplements. Izmir: Izmir Institute of Technology.

[6] Leonard, W. W. (1966). Small modules. Proc. Amer. Math. Soc. 17:527-531.

[7] Maclane, S. (1963). Homology. Chicago Lectures in Mathematics. Chicago-London: Springer-Verlag. 
[8] Mishina, A. P., Skornyakov, L. A. (1960). Abelian Groups and Modules. ChicagoLondon: Amer. Mathematical Society.

[9] Nunke, R. J. (1962). Purity and Subfunctor of the Identity. Topics in Abelian groups. Proc. Sympos., New Mexico State Univ. Vol. 1, pp. 121-171.

[10] Pancar, A. (1997). Generation of proper classes of short exact sequences. Internat. J. Math. and Math. Sci. 20:465-474.

[11] Zöschinger, H. (1974). Komplementierte Moduln über Dedekindringen. J. Alg. 29:42-56.

[12] Zöschinger, H. (1974). Moduln die in jeder Erweiterung ein Komplement haben. Math. Scand. 35:267-287.

[13] Zöschinger, H. (1978). Über torsions- und $\kappa$-elemente von $\operatorname{Ext}(C, A)$. Journal of Algebra 50:299-336.

[14] Zöschinger, H. (1978). Invarianten wesentlicher Überdeckungen. Math. Ann. 237:193-202. 